\title{
A Real-World Study of Prognosis of NOMO Hepatocellular Carcinoma with Hepatic Resection Based on SEER Database
}

\author{
Guangxi Zhu $\mathbb{D}^{D}$, Wensheng Wang $\mathbb{D}^{\mathbb{D}}$, Qin Liu, Dongfeng Chen $\mathbb{D}$, and Liangzhi Wen \\ Department of Gastroenterology, Daping Hospital, Army Medical University, Chongqing, China \\ Correspondence should be addressed to Dongfeng Chen; chendf1981@126.com and Liangzhi Wen; wenliangzhi@126.com
}

Received 10 December 2019; Revised 1 March 2020; Accepted 10 March 2020; Published 1 April 2020

Academic Editor: Niccola Funel

Copyright (c) 2020 Guangxi Zhu et al. This is an open access article distributed under the Creative Commons Attribution License, which permits unrestricted use, distribution, and reproduction in any medium, provided the original work is properly cited.

\begin{abstract}
Aim. To develop and validate a simple-to-use nomogram for prediction of 3-/5-year survival in patients with N0M0 hepatocellular carcinoma after curative liver resection. Patients and Methods. Patients diagnosed HCC with hepatic resection in the Surveillance, Epidemiology, and End Results (SEER) database were included to identify prognostic factors of overall survival. Multivariate Cox regression were used to create a nomogram. Results. We identified $4856 \mathrm{HCC}$ with hepatic resection from the SEER database. A nomogram to predict long-term survival with a C-index 0.667 (95\% CI, 0.653 to 0.681 ) is more efficient than TNM staging with a lower C-index 0.613 (95\% CI, 0.597 to 0.629). The C-index was confirmed to be 0.663 (95\% CI, 0.640 to 0.686 ) through validation, suggesting a good discrimination and a good prediction capability. Conclusions. The nomogram is a simple and effective screening tool for assessing the prognosis of HCC with hepatic resection and assists with the planning of individual postoperative surveillance protocols.
\end{abstract}

\section{Introduction}

Hepatocellular carcinoma (HCC) is one of the most common and most malignant tumors, with seventh incidence rate and second cancer-related mortality rate worldwide [1], while both rates are ranked third in China [2]. To date, surgical treatment is the best treatment for HCC patients without regional lymph node metastasis and distant metastasis, and hepatic resection remains the best therapeutic option for their potential curative outcomes, which can significantly prolong their survival. But around 50\% of these patients suffered relapse within 2 years after surgery. Let alone a large number of patients have already lost their chance of surgery at the time of diagnosis [3]. However, there are currently not many reports on the effects of hepatic resection, and also a lack of effective methods for assessing the patients' prognosis. In general, TNM staging is strongly correlated with survival, and the American Joint Committee on Cancer (AJCC)just released its 8th edition [4]. Nevertheless, different prognosis could still be observed at the same stage according to TNM staging system. The difference may be due to other prognostic factors such as age, sex, etc. Therefore, a more refined method for predicting individualized survival of HCC is in needed, and a nomogram is such a good method to serve this purpose. Nomograms have been designed to serve in many different human cancers, and they have all shown a good advantage over other traditional staging systems [5-7]. The Surveillance, Epidemiology, and End Results (SEER) database is an important resource that based on American people for understanding the impact of pathological diagnosis on population groups [8]. The purpose of our study was to perform a Nomogram analysis and validation of the prognosis of surgically treated HCC patients based on the SEER database.

\section{Patients and Methods}

2.1. Data Source and Study Cohort. We visited the SEER database (https://seer.cancer.gov/) and used SEERStat8.3.5 software to obtain online information on hepatocellular carcinoma patients (from 1973 to 2015) and related prognostic information (site recode ICD-O-3/WHO 2008 = Liver). A total of 126024 cases were viewed, and 4856 met the inclusion criteria: (1) patients with clear histopathological diagnosis 
TABle 1: Demographics and clinicopathologic characteristics of patients with HCC (Abbreviations: Surg_site: surgery of primary site; AFP: the highest serum $\alpha$-fetoprotein level prior to treatment; Scope_Reg-LN: scope of regional lymph node surgery; Marital_status: marital status at diagnosis; API: Asian or Pacific Islander; AI/AN: American Indian/Alaska Native).

\begin{tabular}{|c|c|c|c|c|c|}
\hline \multirow{2}{*}{ Demographic character } & \multicolumn{2}{|c|}{ Derivation cohort $(n=3400)$} & \multicolumn{2}{|c|}{ Validation cohort $(n=1456)$} & \multirow{2}{*}{$P$} \\
\hline & No. of patients & $\%$ & No. of patients & $\%$ & \\
\hline Age & & & & & 0.158 \\
\hline$\leq 55$ & 730 & 21.5 & 311 & 21.4 & \\
\hline$\leq 65$ & 1186 & 34.9 & 467 & 32.1 & \\
\hline$\leq 75$ & 1008 & 29.6 & 474 & 32.6 & \\
\hline $75+$ & 476 & 14.0 & 204 & 14.0 & \\
\hline Race & & & & & 0.500 \\
\hline White & 1947 & 57.3 & 831 & 57.1 & \\
\hline Black & 429 & 12.6 & 182 & 12.5 & \\
\hline API & 985 & 29.0 & 433 & 29.7 & \\
\hline $\mathrm{AI} / \mathrm{AN}$ & 39 & 1.1 & 10 & 0.7 & \\
\hline \multicolumn{6}{|l|}{ Sex } \\
\hline Male & 2426 & 71.4 & 1034 & 71.0 & \\
\hline Female & 974 & 28.6 & 422 & 29.0 & \\
\hline Grade & & & & & 0.832 \\
\hline I & 700 & 20.6 & 309 & 21.2 & \\
\hline II & 1855 & 54.6 & 802 & 55.1 & \\
\hline III & 773 & 22.7 & 314 & 21.6 & \\
\hline IV & 72 & 2.1 & 31 & 2.1 & \\
\hline AJCC_8 & & & & & 0.803 \\
\hline IA & 212 & 6.2 & 93 & 6.4 & \\
\hline IB & 1706 & 50.2 & 738 & 50.7 & \\
\hline II & 866 & 25.5 & 359 & 24.7 & \\
\hline $\operatorname{III}(\mathrm{A} / \mathrm{B})$ & 215 & 6.3 & 80 & 5.5 & \\
\hline IIIA & 205 & 6.0 & 95 & 6.5 & \\
\hline IIIB & 196 & 5.8 & 91 & 6.3 & \\
\hline Tumor_size & & & & & 0.328 \\
\hline$\leq 5 \mathrm{~cm}$ & 1738 & 51.1 & 778 & 53.4 & \\
\hline$\leq 10 \mathrm{~cm}$ & 1093 & 32.1 & 443 & 30.4 & \\
\hline $10+\mathrm{cm}$ & 569 & 16.7 & 235 & 16.1 & \\
\hline Surg_site & & & & & 0.155 \\
\hline Wedge/segment & 1936 & 56.9 & 849 & 58.3 & \\
\hline Lobectomy & 1170 & 34.4 & 505 & 34.7 & \\
\hline Extend-lobectomy & 294 & 8.6 & 102 & 7.0 & \\
\hline Scope_Reg-LN & & 0.0 & & & 0.343 \\
\hline Yes & 464 & 13.6 & 184 & 12.6 & \\
\hline None/unknown & 2936 & 86.4 & 1272 & 87.4 & \\
\hline Radiation & & & & & 0.757 \\
\hline Yes & 70 & 2.1 & 32 & 2.2 & \\
\hline No & 3330 & 97.9 & 1424 & 97.8 & \\
\hline Chemotherapy & & & & & 0.479 \\
\hline Yes & 436 & 12.8 & 176 & 12.1 & \\
\hline No/unknown & 2964 & 87.2 & 1280 & 87.9 & \\
\hline AFP & & & & & 0.318 \\
\hline Positive & 1589 & 46.7 & 676 & 46.4 & \\
\hline Negative & 973 & 28.6 & 444 & 30.5 & \\
\hline Unknown & 838 & 24.6 & 336 & 23.1 & \\
\hline
\end{tabular}


TABLe 1: Continued.

\begin{tabular}{|c|c|c|c|c|c|}
\hline \multirow{2}{*}{ Demographic character } & \multicolumn{2}{|c|}{ Derivation cohort $(n=3400)$} & \multicolumn{2}{|c|}{ Validation cohort $(n=1456)$} & \multirow{2}{*}{$P$} \\
\hline & No. of patients & $\%$ & No. of patients & $\%$ & \\
\hline Ishak_score & & & & & 0.713 \\
\hline F0 & 668 & 19.6 & 301 & 20.7 & \\
\hline F1 & 551 & 16.2 & 234 & 16.1 & \\
\hline Unknown & 2181 & 64.1 & 921 & 63.3 & \\
\hline Marital_status & & & & & 0.434 \\
\hline Married & 2079 & 61.1 & 898 & 61.7 & \\
\hline Never & 535 & 15.7 & 248 & 17.0 & \\
\hline Ever & 661 & 19.4 & 258 & 17.7 & \\
\hline Unknown & 125 & 3.7 & 52 & 3.6 & \\
\hline
\end{tabular}

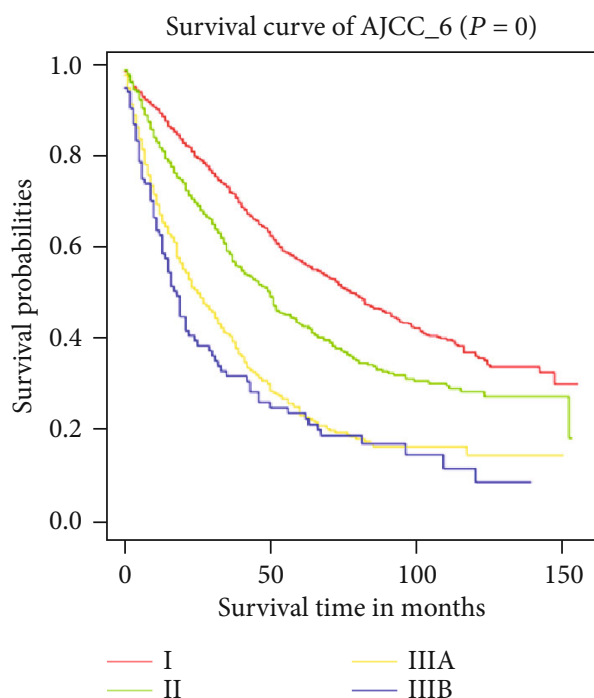

(a)

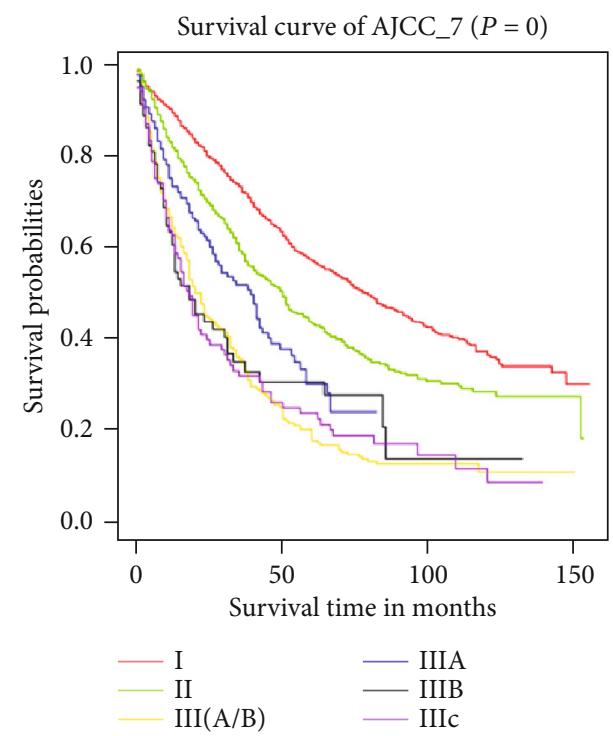

(b)

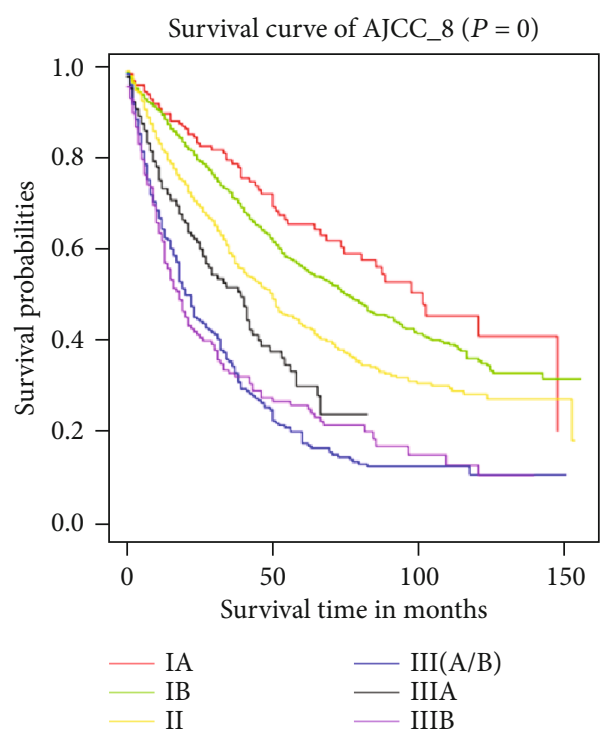

(c)

Figure 1: Kaplan-Meier survival curves of the derivation cohorts generated for different American Joint Committee on Cancer (AJCC) editions. (a-c) AJCC sixth, seventh, and eighth edition, respectively. 

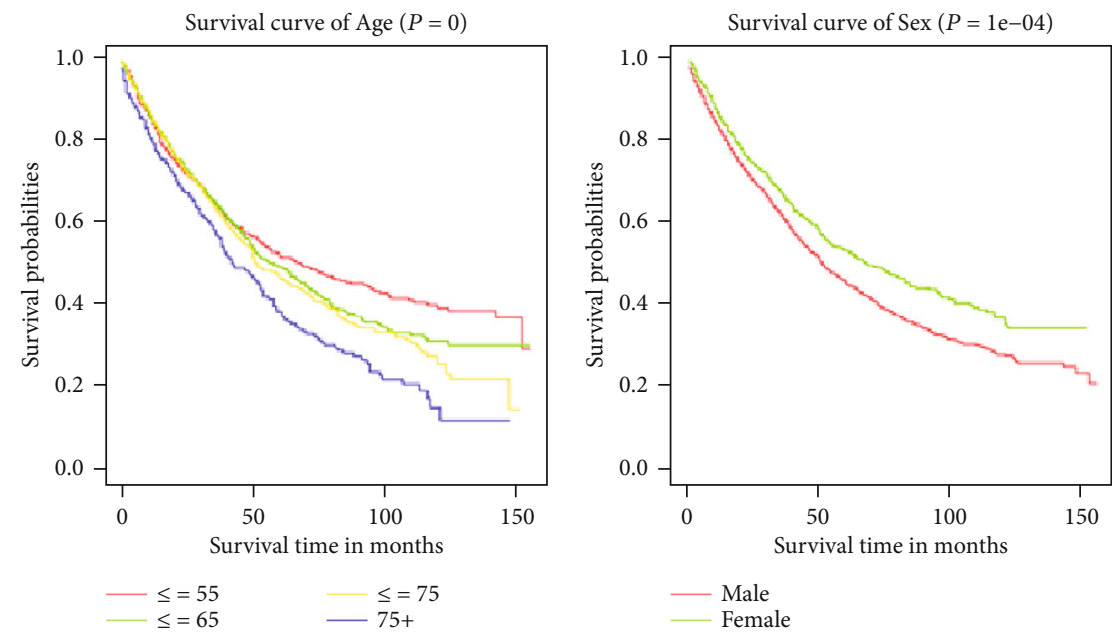

(a)

(b)
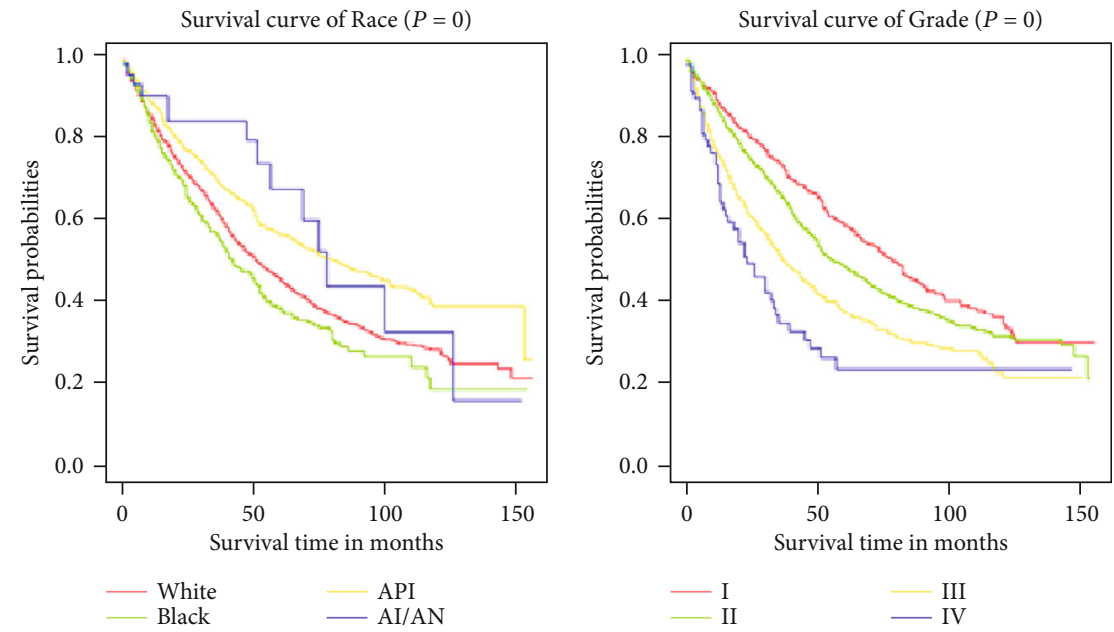

(c)

(d)
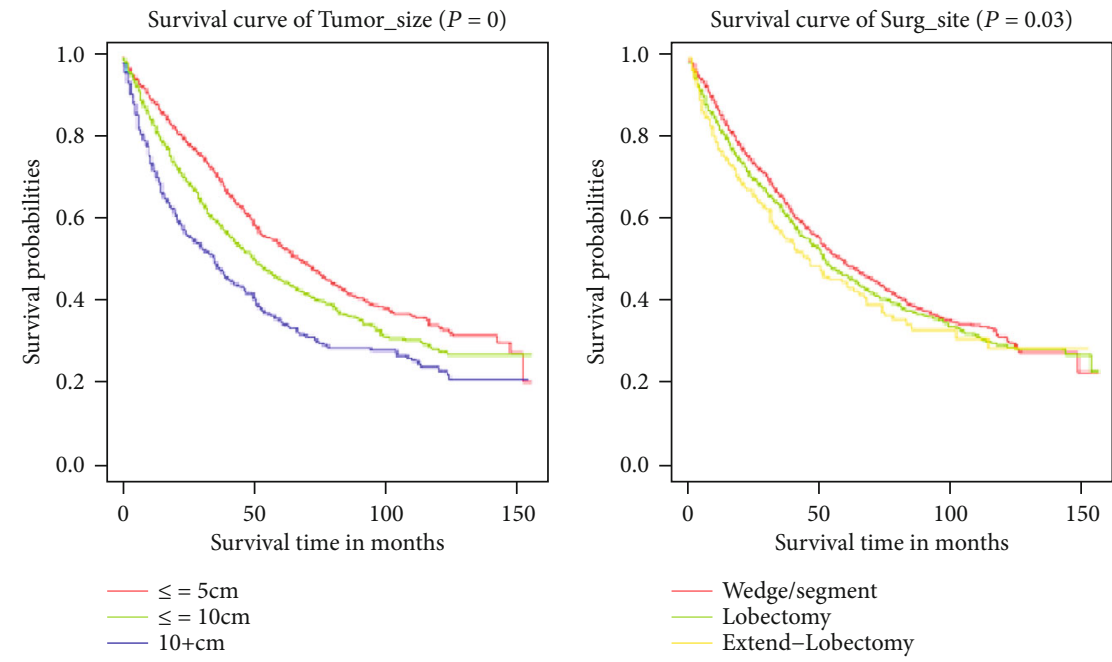

(e)

(f)

Figure 2: Continued. 

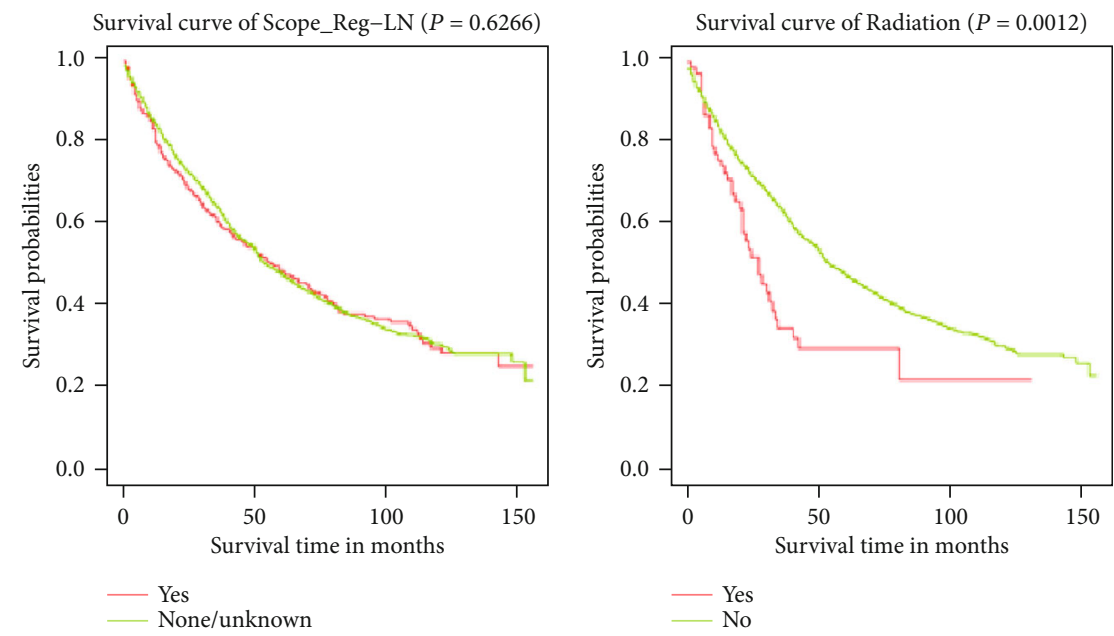

(g)

(h)
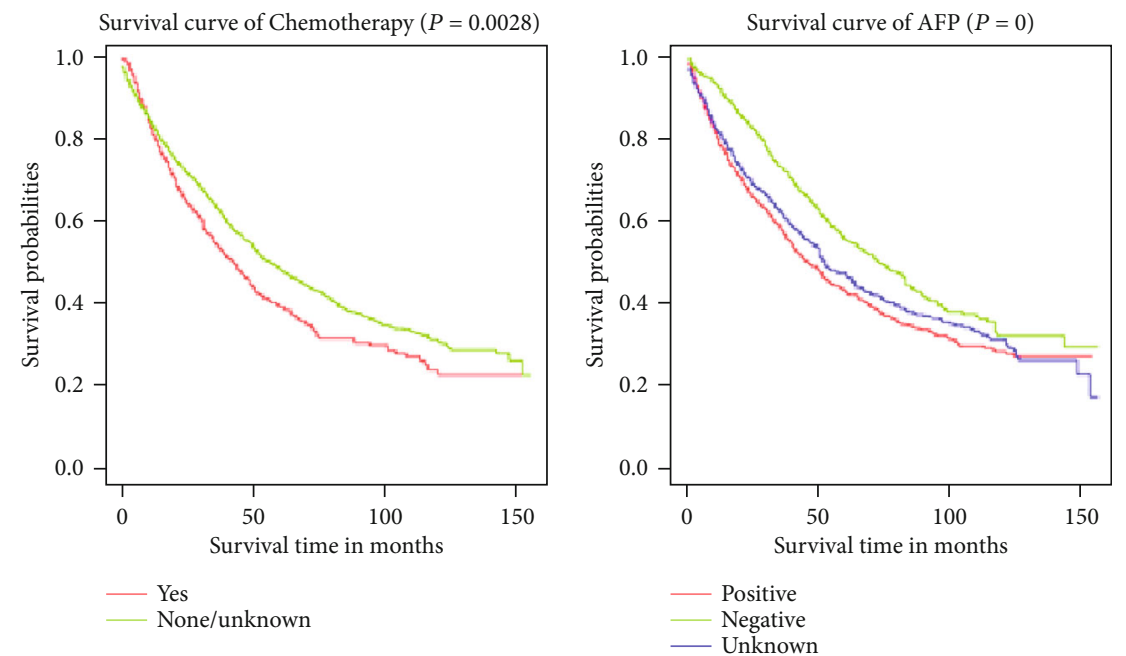

(i)

(j)

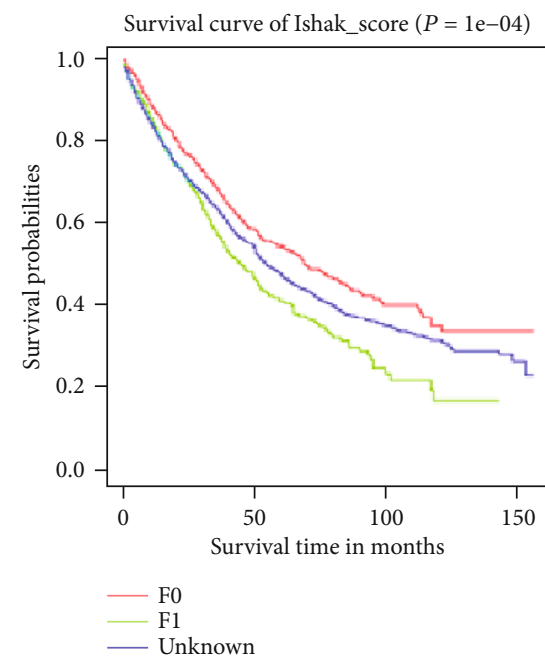

(k)

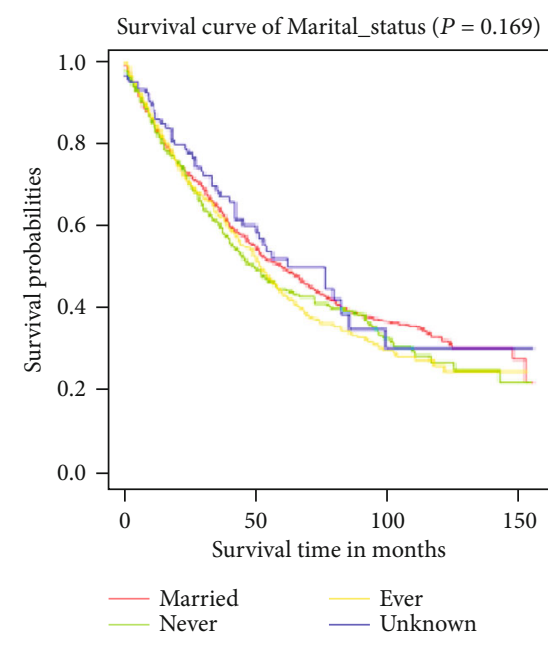

(1)

FIgURE 2: Kaplan-Meier survival curves of the derivation cohorts generated for relative risk factors. (a-l) Possible correlations between Survival and 13 risk factors (Age, Sex, Race, Grade, AJCC_8, Tumor_size, Surg_size, Scope_Reg-LN, Radiation, Chemotherapy, AFP, Ishak_score, and Marital_status). All using univariate Cox analysis. 


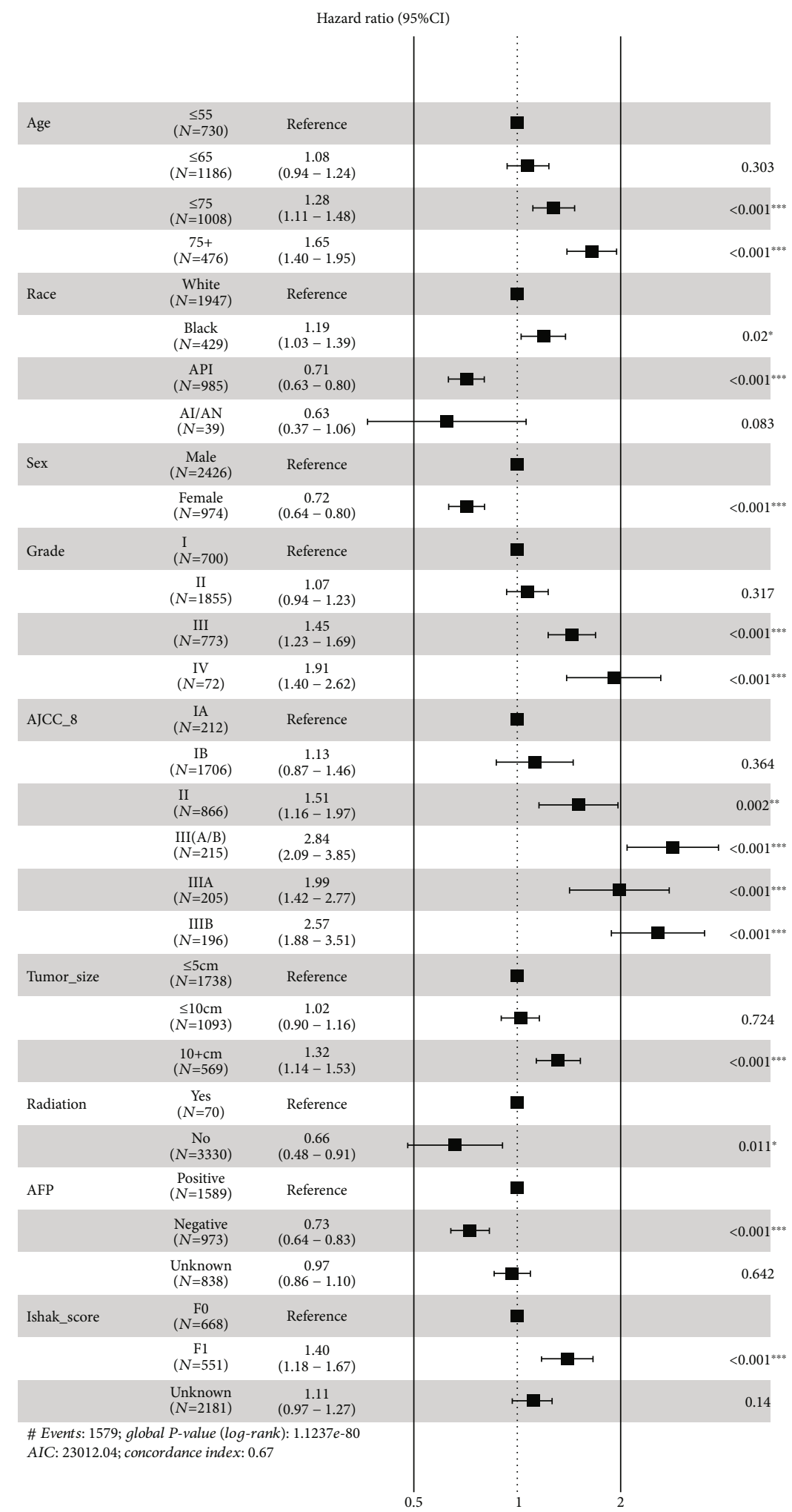

FIGURE 3: Multivariate analysis of the derivation cohort.

(confirmed as positive histology); (2) patients with tissue differentiation grade I, II, III, or IV based on ICD-O-2 criteria; and (3) no regional lymph node metastasis or extrahepatic metastasis (N0M0). We enrolled these 4856 patients in this present study, including 2254 patients who died at the end of the follow-up. The mean follow-up of these 4856 HCC patients is 38 months.

2.2. Nomogram Construction and Validation. For nomogram construction and validation, we randomly assigned $70 \%$ of 


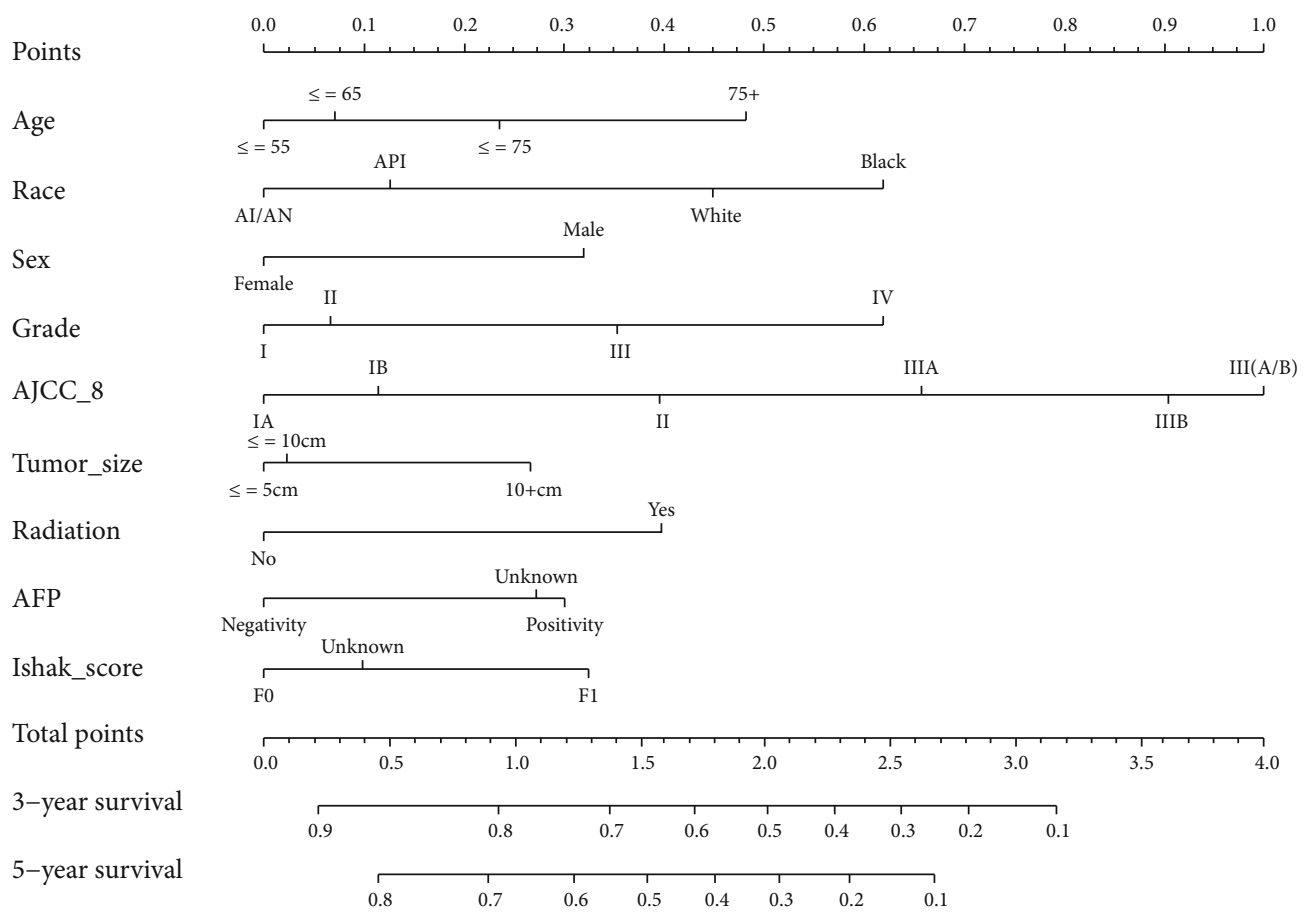

FIGURE 4: Nomogram predicting 3-/5-year survival probability of HCC patients after hepatectomy. Total points are gained by adding up all the points of each variable, and the vertical projections on lower axis present the 3-/5-year survival probability of HCC patients after hepatectomy. AFP: the highest serum $\alpha$-fetoprotein level prior to treatment.

the patients to the derivation cohort $(n=3400)$ and $30 \%$ to validation cohort $(n=1456)$. Cox proportional hazard models were used to assess the univariate and multivariate analyses of the risk factors associated with patient survival. The construction of survival nomogram was based on the multivariate analysis. Hazard ratios are presented with $95 \%$ CI. The nomogram was validated using the concordance index (C-index) and calibration plots. The C-index measures the probability of concordance between predicted and observed survival, similar to the area under the receiver operating characteristic (ROC) curve for censored data. A calibration plot was used to assess the prediction accuracy of the nomogram by plotting the actual survival against the nomogram-predicted survival probabilities. The AJCC system, as one of the important risk factors, was specially pointed out in this study for it has been widely used; we compared the three editions (6th, 7th, and 8th) of AJCC system and proved that the 8 th version overweighed the others, so we chose 8th AJCC to compare with our nomogram for their effectiveness on predicting the prognosis of HCC patients. As no personal confirmation information was contained in this present study, and all the datasets used were public and available online, so personal informed consent and ethical approval are not required.

2.3. Statistical Analysis. Variables used in this study include vital status, survival months, age, race, sex, tumor differentiation grade, tumor size, AJCC staging system, surgery of primary site, radiation, chemotherapy, level of $\alpha$-fetoprotein prior to treatment, AJCC classifies fibrosis scores (also called
Ishak score), and marital status. Excel 2007 was used to organize the data. The statistical analyses were performed using $\mathrm{R}$ software version 3.5.2 (https://www.r-project.org) with the survival and design packages (rms, caret, ROCR, rmda, and survivalROC). Overall survival (OS) was calculated from diagnosis to death from any cause. The Kaplan-Meier method and log-rank test were used to evaluate OS differences. The significant level was set at 0.05 , and all tests were two sided.

\section{Results}

3.1. Clinical Characteristics of Patients in Derivation and Validation Cohort. The clinical characteristics of HCC patients after hepatectomy in derivation cohort $(n=3400)$ and validation cohort $(n=1456)$ are listed in Table 1 . Total 13 categorical variables were selected to reflect these patients' demographics and clinicopathologic conditions. And no significant differences were found among these variables between the derivation cohort and validation cohort $(P>0.05$ in all cases).

3.2. Evaluation of Three AJCC Editions. In order to compare the accuracy of three AJCC editions (6/7/8) for predicting the prognosis of HCC patients after hepatectomy, Kaplan-Meier curves were formed for each of the staging edition. The results are shown in Figure 1. Although all the curves displayed clear prognostic stratification, some overlapping was observed between the survival curves of stages IIIA and IIIB in AJCC sixth edition (Figure 1(a)), the same as stages IIIA, IIIB, and IIIC in the seventh edition (Figure 1(b)). While 


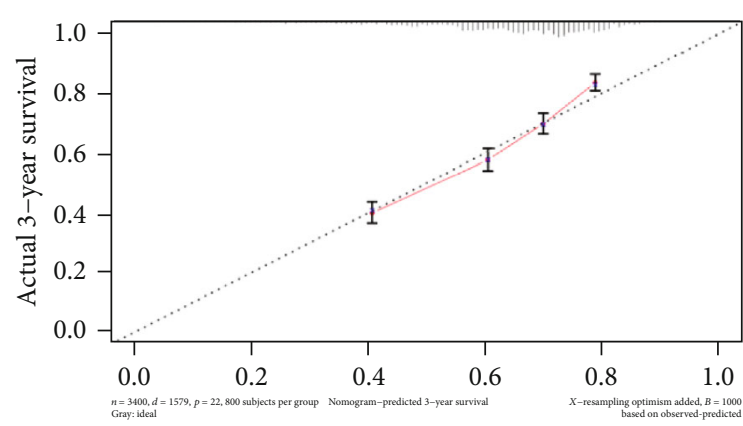

(a)

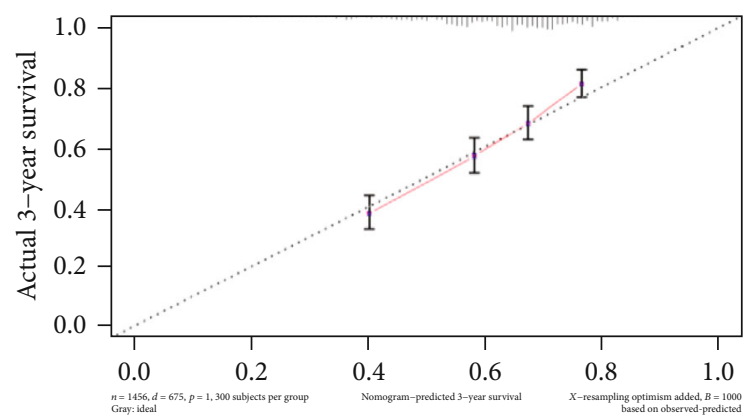

(c)

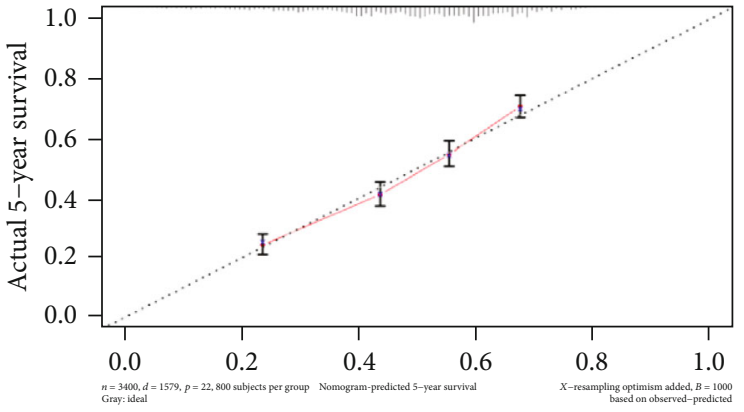

(b)

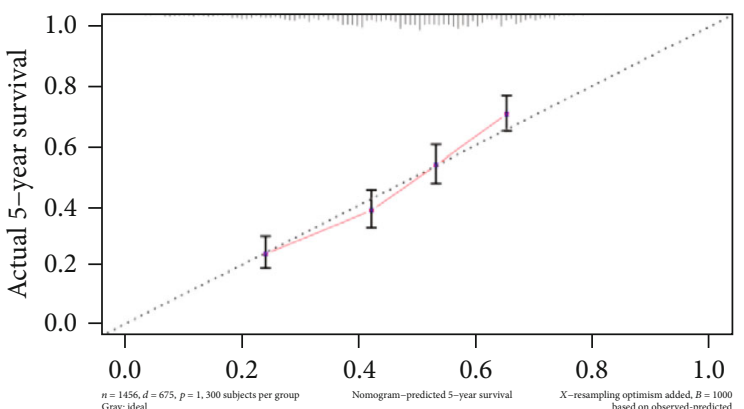

(d)

FIgURE 5: Calibration plot of the nomogram in the derivation cohort and validation cohort. The $x$-axis is the predicted survival calculated by the nomogram, and the $y$-axis is the actual survival estimated by the Kaplan-Meier method.

no overlapping was observed in the eighth edition (Figure 1(c)), except for the survival curve of III(A/B). Considering stage III(A/B) itself could not make clear staging differences from IIIA and IIIB, the AJCC eighth edition displayed higher accuracy than the other two editions by clearly distinguishing the prognosis of HCC patients with different stages.

3.3. Prognostic Nomogram for Overall Survival (OS). The correlations between survival and 13 categorical variables were evaluated by univariate Cox analysis for the patients in the derivation cohort. The results are listed in Figure 2. In which variables with a significant $P$ value $(P<0.05)$ were further enrolled in multivariate Cox regression to identify the potential independent risk factors of overall survival. The results of multivariate analyses of survival are listed in Figure 3, which identified Age, Race, Sex, Grade, AJCC_8, Tumor_size, Radiation, level of $\alpha$-fetoprotein prior to treatment (AFP), and Ishak_score as independent risk factors of OS.

The prognostic nomogram was established by integrating all the nine independent factors closely related to OS in the derivation cohort (Figure 4). The C-index of the established nomogram for OS prediction was 0.667 (95\% CI, 0.653 to 0.681 ) and was confirmed to be 0.663 (95\% CI, 0.640 to $0.686)$ through validation cohort verification, which suggested good discrimination of our model. The calibration plot showed a good agreement between the actual outcomes and nomogram prediction in the probability of 3-/5-year survival (Figures 5(a) and 5(b)). And the calibration plot of the nomogram in the validation cohort is similar to the deriva- tion cohort, which suggests that the nomogram based on derivation cohort is valid and stable (Figures 5(c) and 5(d)).

3.4. Comparison of Accuracy for OS Prediction between Nomogram and AJCC Staging System. It has been proved that AJCC eighth edition has significant advantages over other AJCC editions for predicting overall survival. Therefore, for comparison, the C-index of the AJCC8 staging system for survival prediction was 0.613 (95\% CI, 0.597 to 0.629$)$, both of the C-index for the nomogram in derivation cohort (0.667, 95\% CI, 0.653 to 0.681 ) and validation Cohort $(0.663,95 \%$ CI, 0.640 to 0.686$)$ were significantly higher than that of the AJCC8 $(P<0.05)$.

3.5. Risk Stratification of the Nomogram. To determine the performance of the established nomogram in stratifying risk of HCC patients, we defined those with a risk score higher than the median in the derivation cohorts as high risk, otherwise, defined as low risk (Figure 6(a)). The survival curves generated according to the nomogram-based high and low risk stratification were shown in Figure 6(c). The survival times were significantly differentiated between these two subgroups (Figures 6(b) and 6(c), $P=0$ ).

\section{Discussion}

Our study proposed a risk prediction model for predicting OS of HCC patients after hepatic resection. After certification, the $\mathrm{C}$-index indicates that the prognostic value of this current nomogram was superior to AJCC staging system; it provides patients and health workers with a more 


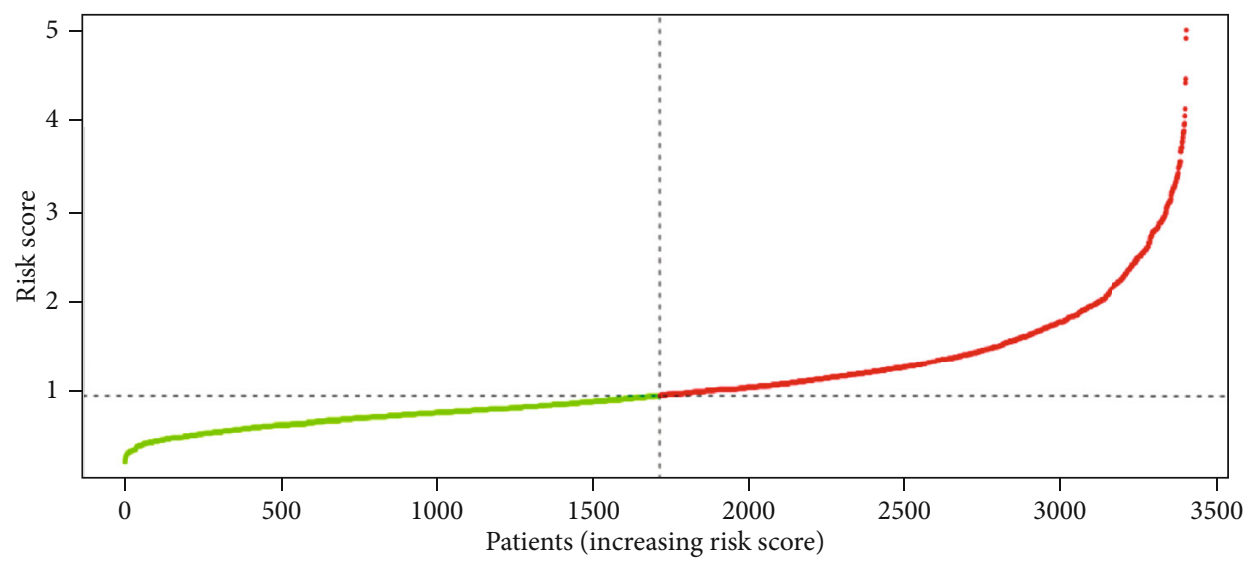

- High risk

- Low risk

(a)

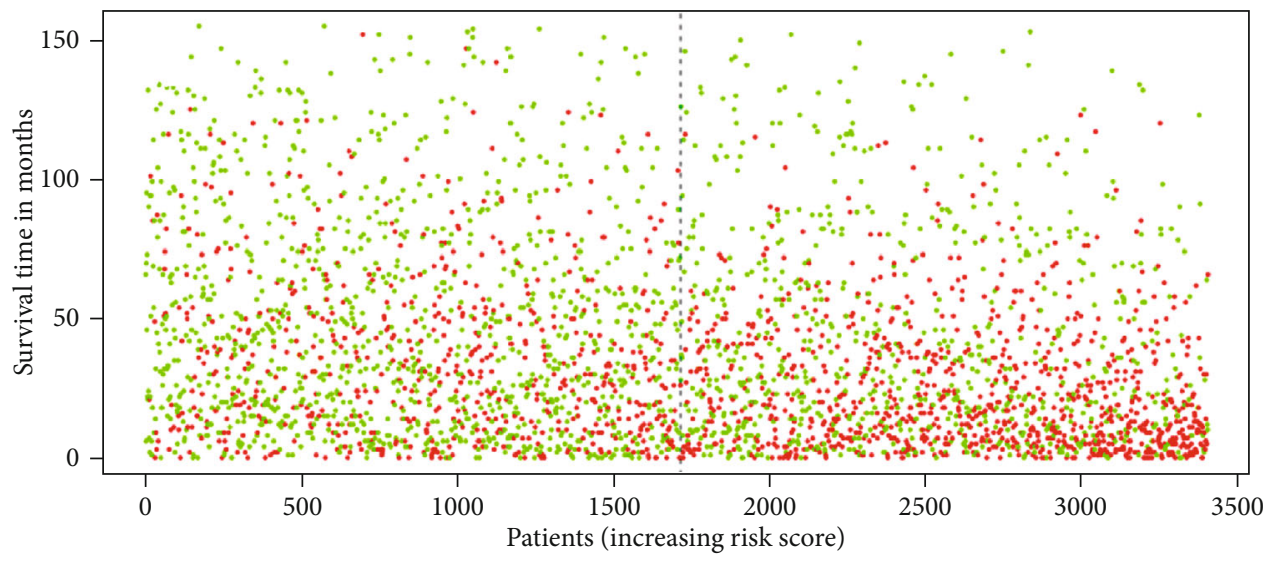

- Dead

- Alive

(b)

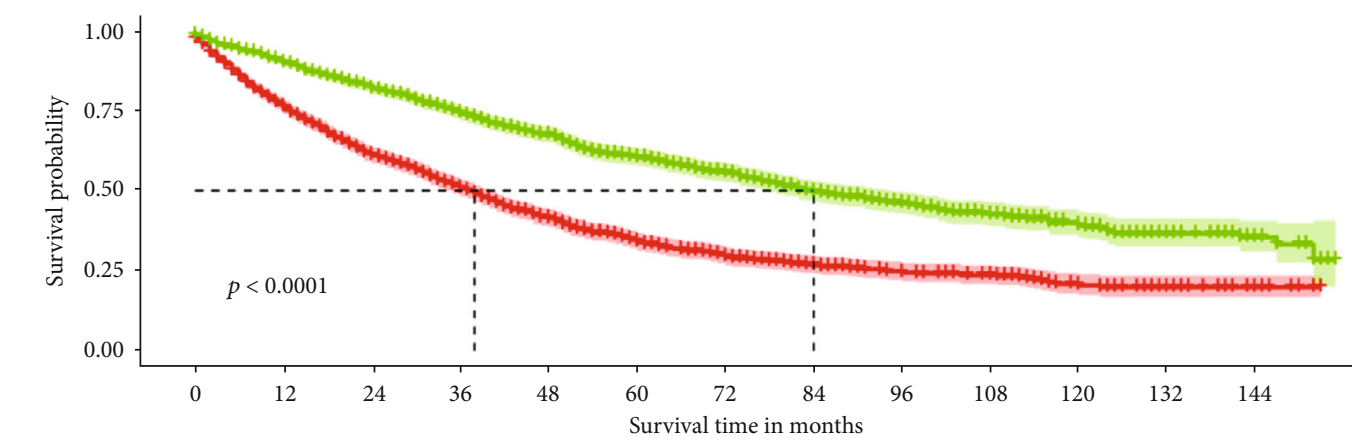

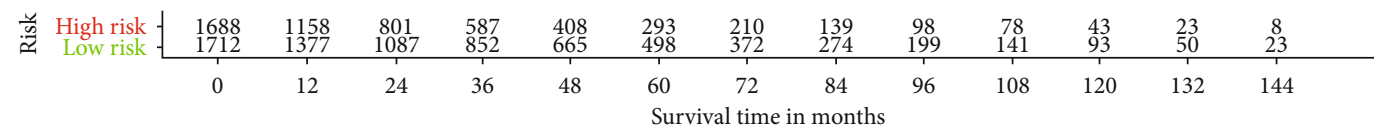
Risk

+ High risk

+ Low risk

(c)

FIGURE 6: Kaplan-Meier survival curves of HCC patients with high risk scores versus low risk scores. 
convenient and friendly method to predict postoperative life expectancy. The nomogram integrated nine independent risk factors of OS of these patients to generate a total risk score that could be converted into 3-/5-year postoperative survival probability.

Based on this model, the older, black, and male patients share the higher risk of shorter survival. To the best of our knowledge, the advanced tumor grade is associated with a poor overall survival [9]. We analyzed the potential risk of advanced tumor grade; it turns out that compared with grade 1 , advanced grades had much higher risk of poor prognosis (HR: 1.45 [CI: 1.23-1.69] for grade 3; HR: 1.91 [CI: 1.402.62] for grade 4). Tumor size is considered to be an important prognostic indicator of overall survival of postoperative HCC patients, also recognized as one of the main factors influencing the prognostic management of $\operatorname{HCC}[10,11]$. There is nearly no doubt that the outcomes are much better for small tumor $(\leq 5 \mathrm{~cm})$ [12]; however, some studies have also shown that the prognosis of excising larger tumors $(>5 \mathrm{~cm})$ is also promising [13]. In this study, tumor size was verified to be an independent risk factor for OS, with a HR of 1.32 (CI: 1.14-1.53) with a size over $10 \mathrm{~cm}$, compared with those smaller than $5 \mathrm{~cm}$.

Radiation therapy plays an important role in relieving tumor burden and delaying the progression of HCC, especially for patients who are no longer suitable for surgery. However, the limitation of radiotherapy is also explicit; since both the tumor tissue and adjacent normal liver tissue are sensitive to radiation, it is inevitable to cause some unexpected damage [14]. Recent study found that proton beam therapy (PBT) can make compensation to this unbiased damaging process. PBT can not only reduce radiation-related hepatotoxicity but also enhance sensitivity of tumor tissue to radiation dose [15]. According to this study, HCC patients who underwent radiation therapy had higher risk of suffering poorer OS, with a HR of 1.51 (CI: 1.10-2.08), which suggests traditional radiation therapy as an independent adverse prognostic factor of OS.

$\alpha$-Fetoprotein (AFP) is often reported to be elevated in HCC patients accompanied with large tumor, early tumor recurrence, and vascular invasion [16]. Previous studies have shown that preoperative or postoperative AFP levels were highly correlated with the prognosis of HCC after hepatic resection. HCC patients with preoperative serum AFP $\leq 20 \mathrm{ng} / \mathrm{mL}$ and without surgical contraindications predict better prognosis after surgical treatment (compared with AFP $>20 \mathrm{ng} / \mathrm{mL}$ ) [17]. Silva et al. underlined the importance of baseline AFP levels in HCC: as baseline AFP levels increase in unadjusted populations, the median overall survival of HCC patients dramatically decreases. And considering AFP as a continuous variable rather than a categorical one helps to further understand the correlation between baseline AFP levels and overall survival of HCC patients [18]. In our study, we verified AFP as an independent risk factor of postoperative survival of HCC patients (HR: 1.37, CI: 1.20-1.56). Considering the inconsistency of studies about AFP as a useful prognostic factor, for example, Giannini et al. found that AFP did not play an important role in predicting the prognosis of small hepatocellular carcinoma [19]. Therefore, AFP just took up a small proportion in the total points of nomogram. Further studies are urgently needed to explore the role of AFP in HCC prognosis.

In this study, calibration plots showed consistency between the predict and actual survival, which ensured the accuracy and reliability of the prognostic nomogram. According to the nomogram, HCC patients can be divided into high-risk and low-risk subgroups, which indicate significantly different overall survival rates. The main benefits of this study lies in the following aspects. First, the nomogram is based on factors available in the patient's preoperative assessment and can assists with the planning of individual postoperative surveillance protocols of HCC patients. Second, SEER database is a large public database which can provide amounts of case samples. This SEER-based study belongs to a real-world research. Third, this nomogram integrates several prognosis-related clinicopathologic factors and have been verified to work more effectively than AJCC staging system alone, which can provide a doctor-patient friendly and effective screening tool for assessing the prognosis of HCC with hepatic resection. Nevertheless, this study also has limitations, with SEER database mainly based on American population, to some extent, regional and racial differences might affect the final outcomes. And there are other factors that may also affect HCC prognosis, which are not included in this current study. In the next step, we need to find out and put more available and potential factors into further research.

\section{Data Availability}

All data or model used during the study are available from the corresponding author by request.

\section{Conflicts of Interest}

The authors declare that they have no conflicts of interest.

\section{Authors' Contributions}

Dongfeng Chen, Liangzhi Wen, and Guangxi Zhu designed the present study. Guangxi Zhu and Wensheng Wang performed the data analysis and interpretation. Guangxi Zhu, Wensheng Wang, and Liangzhi Wen wrote the manuscript. All authors approved the final manuscript. Guangxi Zhu and Wensheng Wang contributed equally to this work.

\section{Acknowledgments}

This study was supported by National Natural Science Foundation of China (NCFS:81802459) and Chongqing Natural Science Foundation (cstc2018jcyjAX0603).

\section{References}

[1] F. Bray, J. Ferlay, I. Soerjomataram, R. L. Siegel, L. A. Torre, and A. Jemal, "Global cancer statistics 2018: Globocan estimates of incidence and mortality worldwide for 36 cancers in 185 countries," CA: A Cancer Journal for Clinicians, vol. 68, no. 6, pp. 394-424, 2018. 
[2] W. Chen, R. Zheng, P. D. Baade et al., "Cancer statistics in China, 2015," CA: A Cancer Journal for Clinicians, vol. 66, no. 2, pp. 115-132, 2016.

[3] M. Maluccio and A. Covey, "Recent progress in understanding, diagnosing, and treating hepatocellular carcinoma," $C A$ : A Cancer Journal for Clinicians, vol. 62, no. 6, pp. 394-399, 2012.

[4] S. K. Kamarajah, T. L. Frankel, C. Sonnenday, C. S. Cho, and H. Nathan, "Critical evaluation of the American Joint Commission on Cancer (AJCC) 8th edition staging system for patients with hepatocellular carcinoma (HCC): a Surveillance, Epidemiology, End Results (SEER) analysis," Journal of Surgical Oncology, vol. 117, no. 4, pp. 644-650, 2018.

[5] D. S. Han, Y. S. Suh, S. H. Kong et al., "Nomogram predicting long-term survival after d2 gastrectomy for gastric cancer," Journal of Clinical Oncology, vol. 30, no. 31, pp. 3834-3840, 2012.

[6] P. G. Rose, J. Java, C. W. Whitney et al., "Nomograms predicting progression-free survival, overall survival, and pelvic recurrence in locally advanced cervical cancer developed from an analysis of identifiable prognostic factors in patients from nrg oncology/gynecologic oncology group randomized trials of chemoradiotherapy," Journal of Clinical Oncology, vol. 33, no. 19, pp. 2136-2142, 2015.

[7] W. Liang, L. Zhang, G. Jiang et al., "Development and validation of a nomogram for predicting survival in patients with resected non-small-cell lung cancer," Journal of Clinical Oncology, vol. 33, no. 8, pp. 861-869, 2015.

[8] M. A. Duggan, W. F. Anderson, S. Altekruse, L. Penberthy, and M. E. Sherman, "The Surveillance, Epidemiology, and End Results (SEER) program and pathology: toward strengthening the critical relationship," The American Journal of Surgical Pathology, vol. 40, no. 12, pp. e94-e102, 2016.

[9] J. D. Wayne, G. Y. Lauwers, I. Ikai et al., "Preoperative predictors of survival after resection of small hepatocellular carcinomas," Annals of Surgery, vol. 235, no. 5, pp. 722-731, 2002.

[10] V. S. Yip, D. Gomez, C. Y. Tan et al., "Tumour size and differentiation predict survival after liver resection for hepatocellular carcinoma arising from non-cirrhotic and non-fibrotic liver: a case-controlled study," International Journal of Surgery, vol. 11, no. 10, pp. 1078-1082, 2013.

[11] C.-N. Yeh, M.-F. U. Chen, W.-C. Lee, and L.-B. Jeng, "Prognostic factors of hepatic resection for hepatocellular carcinoma with cirrhosis: univariate and multivariate analysis," Journal of Surgical Oncology, vol. 81, no. 4, pp. 195-202, 2002.

[12] S. Arii, Y. Yamaoka, S. Futagawa et al., "Results of surgical and nonsurgical treatment for small-sized hepatocellular carcinomas: a retrospective and nationwide survey in Japan," Hepatology, vol. 32, no. 6, pp. 1224-1229, 2000.

[13] X.-D. Zhou, Z.-Y. Tang, B.-H. Yang et al., "Experience of 1000 patients who underwent hepatectomy for small hepatocellular carcinoma," Cancer, vol. 91, no. 8, pp. 1479-1486, 2001.

[14] T. Eggert and T. F. Greten, "Current standard and future perspectives in non-surgical therapy for hepatocellular carcinoma," Digestion, vol. 96, no. 1, pp. 1-4, 2017.

[15] R. H. Yeung, T. R. Chapman, S. R. Bowen, and S. Apisarnthanarax, "Proton beam therapy for hepatocellular carcinoma," Expert Review of Anticancer Therapy, vol. 17, no. 10, pp. 911-924, 2017.

[16] S.-Y. Peng, W. J. Chen, P.-L. Lai, Y.-M. Jeng, J.-C. Sheu, and H.-C. Hsu, "High $\alpha$-fetoprotein level correlates with high stage, early recurrence and poor prognosis of hepatocellular carcinoma: significance of hepatitis virus infection, age, p53 and $\beta$-catenin mutations," International Journal of Cancer, vol. 112, no. 1, pp. 44-50, 2004.

[17] W.-j. Ma, H.-y. Wang, and L.-s. Teng, "Correlation analysis of preoperative serum alpha-fetoprotein (AFP) level and prognosis of hepatocellular carcinoma (HCC) after hepatectomy," World Journal of Surgical Oncology, vol. 11, no. 1, p. 212, 2013.

[18] J. P. Silva, R. A. Gorman, N. G. Berger et al., “The prognostic utility of baseline alpha-fetoprotein for hepatocellular carcinoma patients," Journal of Surgical Oncology, vol. 116, no. 7, pp. 831-840, 2017.

[19] E. G. Giannini, S. Marenco, G. Borgonovo et al., "Alpha-fetoprotein has no prognostic role in small hepatocellular carcinoma identified during surveillance in compensated cirrhosis," Hepatology, vol. 56, no. 4, pp. 1371-1379, 2012. 